\title{
Combination of GNSS orbits using variance component estimation
}

\author{
Gustavo Mansur ${ }^{1,2, *}$, Pierre Sakic ${ }^{1}$, Andreas Brack ${ }^{1}$, Benjamin Männel ${ }^{1}$, and Harald \\ Schuh $^{1,2}$ \\ ${ }^{1}$ GFZ German Research Centre for Geosciences, Helmholtz-Zentrum Potsdam, Section \\ 1.1 - Space Geodetic Techniques, Potsdam \\ ${ }^{2}$ Technische Universität Berlin, Institute for Geodesy and Geoinformation Technology, \\ Faculty VI \\ *Correspondence: mansur@gfz-potsdam.de
}

22 December 2020

\begin{abstract}
Nota Bene: This manuscript has been submitted for publication in Journal of Geodesy. Please note that manuscript has yet to be formally accepted for publication. Subsequent versions of this manuscript may have slightly different content. If accepted, the final version of this manuscript will be available via the Peer-reviewed Publication DOI link on the right-hand side of the EarthArXiv webpage. Please feel free to contact any of the authors; we welcome feedback.
\end{abstract}

\begin{abstract}
Over the past years, the International GNSS Service (IGS) has been putting efforts into extending its service towards the Multi-GNSS Experiment and Pilot Project (MGEX). Several MGEX Analysis Centers (ACs) contribute by providing solutions containing not only GPS and GLONASS but also Galileo, BeiDou, and QZSS. The MGEX orbit and clock combination is a product that is still not consolidated inside the IGS and requires studies in order to provide a consistent solution. In this contribution, we present a least-squares framework for a multi-GNSS orbit combination, where the weights used to combine the ACs' orbits are determined by least-squares variance component estimation. We introduce and compare two weighting strategies, where either AC specific weights or AC and constellation specific weights are used. Both strategies are tested using MGEX orbit solutions for a period of two and a half years. They yield similar results with an agreement with the ACs' orbits at the one centimeter level for GPS and up to a few centimeters for the other constellations. The agreement is generally slightly better with the $\mathrm{AC}$ and constellation weighting. A comparison of our combination approach with the official combined IGS final solution using three years of GPS, and GLONASS orbits from the regular IGS processing shows an agreement of better than $5 \mathrm{~mm}$ and $12 \mathrm{~mm}$ for GPS and GLONASS, respectively. An external validation using Satellite Laser Ranging (SLR) is performed for our combined MGEX orbit solutions with both weighting schemes.
\end{abstract}

Keywords: GNSS, MGEX, Variance Components Estimation, Orbit combination

\section{Introduction}

The International GNSS Service (IGS) publishes on a regular basis GPS and GLONASS orbit and clock offset products with the highest accuracy [Johnston et al., 2017]. These final products result 
from a combination using as input the orbits and clock offsets determined by the IGS Analysis Centers (ACs). The motivation for a combination remains the same since the early years of the IGS: to smooth the errors and fill the possible gaps made by ACs during their independent processing, and thus provide a reliable, accurate, and unique product to users [Springer and Beutler, 1993; Beutler et al., 1995]. Such combination is also the best way to have a reference for the comparison and validation of the AC individual results [e.g. Griffiths, 2019].

The method to perform the combination was developed by Springer and Beutler [1993] and described in detail by Kouba et al. [1994]. Some updates were made over the years mainly to improve the clock combination and the alignment with the current ITRF [Kouba and Springer, 2001]. The complete history of changes in the combination strategy is described in the combination summary files' header.

In addition, the Multi-GNSS Experiment and Pilot Project [MGEX, Montenbruck et al., 2017] is working inside the IGS on the extension of the services regarding new systems and has gained attention since more receivers are capable of tracking new signals, and more constellations were declared operational in the last years, e.g., Galileo in 2016 [ESA, 2016] or Beidou in 2018 [CSNO, 2018]. Several ACs are recently putting efforts into extending their products and providing the new constellations in their solutions.

Nevertheless, no official combination for the new constellations is available so far. Inside this perspective, the IGS Analysis Center Coordinator (ACC) proposes since September 2019 an experimental combined MGEX solution ${ }^{1}$ based on an adaptation of the legacy strategy [Sośnica et al., 2020]. The GFZ IGS working group has also been putting efforts to study and provide a dedicated and entirely consistent orbit and clock combination using the solutions provided by the ACs that contribute to the MGEX project. Previous studies were made adapting the IGS combination software [Fritsche, 2016; Mansur et al., 2020; Sakic et al., 2020], and have shown that significant modifications are needed to combine MGEX products, including an improved weighting strategy adapted to the different constellations. Therefore, only an adaptation of the existing software may not be enough to handle the different constellations with different quality of orbit solutions, as well as may not provide a full perspective about the influence that each $\mathrm{AC}$ has on a specific constellation. Aiming to study and discuss a new method for the orbit combination, two different approaches based on variance component estimation are developed. First, considering ACs-only weights and the second, including a joint weighting scheme based on the AC plus constellation. The weights used to compute the final combined orbits are proportional to the inverse of the estimated variance components.

The overall structure of this manuscript contains five sections. In Section 2, the mathematical least-squares framework of our orbit combination is introduced, whereas Section 3 provides a brief explanation of the steps implemented to combine the orbits. Section 4 summarizes the experiments and compares the two methods, and finally, the summary and conclusions are given in Section 5.

\section{Mathematical framework}

The task of the orbit combination is to transform the orbit products of different ACs to a single combined orbit product. In short, the current IGS combination procedure is as follows. It starts with a simple mean of the orbit solutions from all ACs. Then, seven parameter Helmert transformations between the individual orbits and the mean orbit are performed, and the ACs' orbits are aligned to the mean via these transformations. A weight is derived for each AC from the differences of these transformed orbits and the mean orbit, and a new weighted mean orbit is computed. The same steps are repeated once, starting with the weighted mean orbit, finally yielding the combined orbit product.

\footnotetext{
${ }^{1}$ http://acc.igs.org/mgex_experimental.html
} 
In this section, we present a combination approach that is based on the least-squares principle. The combined orbits are then again a weighted mean of the orbits of the ACs, where the weights are proportional to the inverse variances of the ACs' solutions. These unknown variances are estimated by means of Least Squares Variance Component Estimation (LSVCE). The solution to this problem of a joint least-squares estimation of the combined orbits and the variance components is shown in the following.

\subsection{System model}

The input of the orbit combination are the orbits of the ACs. Let the Earth-fixed coordinates of satellite $s \in\{1, \ldots, S\}$ provided by AC $r \in\{1, \ldots, R\}$ at the time $t$ be given by $\underline{x}_{r}^{s}(t) \in \mathbb{R}^{3}$. We define

$$
\underline{x}_{r}(t)=\left[\underline{x}_{r}^{1}(t)^{\mathrm{T}} \cdots \underline{x}_{r}^{S}(t)^{\mathrm{T}}\right]^{\mathrm{T}} \in \mathbb{R}^{3 S}
$$

and

$$
\underline{x}_{r}=\left[\underline{x}_{r}^{\mathrm{T}}\left(t_{1}\right) \ldots \underline{x}_{r}^{\mathrm{T}}\left(t_{P}\right)\right]^{\mathrm{T}} \in \mathbb{R}^{3 S P},
$$

with $t_{p}, p \in\{1, \ldots, P\}$, the time at epoch $p$. The unknown combined orbit vector $x_{\mathrm{c}} \in \mathbb{R}^{3 S P}$ is defined according to $\underline{x}_{r}$. We assume a relation between the AC orbits and the combined orbits based on the linearized Helmert transformation as

$$
\underline{x}_{r}^{s}(t)=H_{r} \underline{x}_{\mathrm{c}}^{s}(t)+\Delta \underline{x}_{r}+\underline{\varepsilon}_{r},
$$

where the AC specific Helmert transformation matrices $H_{r}$ are in the form of

$$
H=\left[\begin{array}{ccc}
\mu & \theta_{z} \mu & -\theta_{y} \mu \\
-\theta_{z} \mu & \mu & \theta_{x} \mu \\
\theta_{y} \mu & -\theta_{x} \mu & \mu
\end{array}\right]
$$

with $\theta_{x}, \theta_{y}, \theta_{z}$, and $\mu$ the rotation and scale parameters, and $\Delta \underline{x}_{r}$ the translation parameters, that align the ACs' orbits and the combined orbits, and $\underline{\varepsilon}_{r} \in \mathbb{R}^{3 S P}$ an additive noise term. To simplify the system model (3), we apply an invertible transformation to the input orbits

$$
\underline{\bar{x}}_{r}^{s}(t)=H_{r}^{-1}\left(\underline{x}_{r}^{s}(t)-\Delta \underline{x}_{r}\right),
$$

so that the final system model can simply be written as

$$
\underbrace{\left[\begin{array}{c}
\bar{x}_{1} \\
\vdots \\
\underline{x}_{R}
\end{array}\right]}_{\underline{\underline{x}}}=\underbrace{\left[\underline{e}_{R} \otimes I_{3 S P}\right]}_{A} \cdot \underline{x}_{\mathrm{c}}+\underbrace{\left[\begin{array}{c}
\bar{\varepsilon}_{1} \\
\vdots \\
\overline{\bar{\varepsilon}}_{R}
\end{array}\right]}_{\underline{\bar{\varepsilon}}},
$$

where $\underline{e}_{R}$ is an $R$-vector of ones, $I_{3 S P}$ an identity matrix of size $3 S P$, and $\otimes$ the Kronecker product. Note that all information is kept through this transformation and that $\underline{\bar{\varepsilon}}_{r}$ are the transformed versions of $\underline{\varepsilon}_{r}$.

For the stochastic model we assume that

$$
\underline{\bar{\varepsilon}}_{r} \sim \mathscr{N}\left(\underline{0}, \sigma_{r}^{2} I_{3 S P}\right),
$$

i.e., there is an $\mathrm{AC}$ specific variance $\sigma_{r}^{2}$ that is common for all coordinates, and there are no correlations among different coordinates within each AC. Assuming uncorrelated AC solutions, we therefore have

$$
\underline{\bar{\varepsilon}} \simeq \mathscr{N}(\underline{0}, Q) \quad \text { with } \quad Q=\sum_{r=1}^{R} \sigma_{r}^{2} Q_{r},
$$


where $\sigma_{r}^{2}$ are the variance components, and $Q_{r}=\operatorname{diag}\left(\underline{c}_{r}\right) \otimes I_{3 S P}$, with $\underline{c}_{r} \in \mathbb{R}^{R}$ a canonical unit vector, the cofactor matrices with $\sum_{r=1}^{R} \sigma_{r}^{2} Q_{r}$ non-negative definite. These AC specific variance components $\sigma_{r}$ lead to AC specific weights for the orbit combination, $c f$. Section 2.2. An extension allowing for $\mathrm{AC}$ and constellation specific weights is presented in Section 2.4.

We can now solve the system model as described in (6) and (8) for the unknown combined orbits $\underline{x}_{\mathrm{c}}$ and the variance components $\sigma_{r}^{2}, r \in\{1 \ldots, R\}$. The estimation follows the LSVCE framework presented in Amiri-Simkooei [2007] and Teunissen and Amiri-Simkooei [2008]. It is noted that for the computation of the Helmert transformation parameters in Eqns. (3)-(5) the unknown combined orbits $\underline{x}_{\mathrm{c}}$ are required so that the above estimation problem is solved iteratively, where the combined orbits can be initialized with a simple mean and then updated in each iteration.

\subsection{Combined orbit solution}

We recall from Eqns. (6)-(8) that our system is of the form $\underline{\bar{x}}=A \underline{x}_{c}+\underline{\bar{\varepsilon}}$, so that the weighted least-squares solution $\hat{\underline{x}}_{c}$ for the combined orbit is given by

$$
\underline{\hat{x}}_{\mathrm{c}}=\left(A^{\mathrm{T}} Q^{-1} A\right)^{-1} A^{\mathrm{T}} Q^{-1} \underline{\bar{x}} .
$$

With the simplifications

$$
\begin{aligned}
A^{\mathrm{T}} Q^{-1} & =\left(\underline{e}_{R}^{\mathrm{T}} \otimes I_{3 S P}\right) \sum_{r=1}^{R}\left(\operatorname{diag}\left(\underline{c}_{r}\right) \otimes I_{3 S P} \frac{1}{\sigma_{r}^{2}}\right) \\
& =\sum_{r=1}^{R} \underline{c}_{r}^{\mathrm{T}} \otimes I_{3 S P} \frac{1}{\sigma_{r}^{2}}
\end{aligned}
$$

and

$$
\begin{aligned}
A^{\mathrm{T}} Q^{-1} A & =\left[\sum_{r=1}^{R} \frac{1}{\sigma_{r}^{2}}\left(\underline{c}_{r}^{\mathrm{T}} \otimes I_{3 S P}\right)\right]\left(\underline{e}_{R}^{\mathrm{T}} \otimes I_{3 S P}\right) \\
& =\left(\sum_{r=1}^{R} \frac{1}{\sigma_{r}^{2}}\right) I_{3 S P}
\end{aligned}
$$

the combined orbit solution from (9) can be rewritten as

$$
\underline{\hat{x}}_{\mathrm{c}}=\frac{1}{\sum_{r=1}^{R} \frac{1}{\sigma_{r}^{2}}} \cdot \sum_{r=1}^{R} \frac{1}{\sigma_{r}^{2}} \cdot \underline{\bar{x}}_{r},
$$

which shows that the combined orbits are indeed a weighted mean of the ACs' orbits. The estimation of the variance components $\sigma_{r}^{2}$ is shown in the next section.

\subsection{Variance component solution}

Let the vector of unknown variance components that define the weights in Eqn. (12) be defined as $\underline{\sigma}=\left[\sigma_{1}^{2}, \ldots, \sigma_{R}^{2}\right]^{\mathrm{T}}$. Following the LSVCE theory presented by Amiri-Simkooei [2007], the least-squares solution $\underline{\hat{\sigma}}$ of the variance components is given by

$$
\underline{\hat{\sigma}}=\left(A_{\mathrm{vh}}^{\mathrm{T}} Q_{\mathrm{vh}}^{-1} A_{\mathrm{vh}}\right)^{-1} A_{\mathrm{vh}}^{\mathrm{T}} Q_{\mathrm{vh}}^{-1} \mathrm{vh}\left(\underline{t t}^{\mathrm{T}}\right),
$$

with $A_{\mathrm{vh}}=\left[\operatorname{vh}\left(B^{\mathrm{T}} Q_{1} B\right), \ldots, \operatorname{vh}\left(B^{\mathrm{T}} Q_{R} B\right)\right], \underline{t}=B^{T} \underline{\bar{x}}$, and the vh operator that transforms a symmetric matrix into a vector, thereby removing the duplicate entries [Teunissen and Amiri-Simkooei, 
2008]. The columns of $B$ span the null space of $A^{\mathrm{T}}$ so that $A^{\mathrm{T}} B=0$, and $Q_{\mathrm{vh}}^{-1}$ is a weight matrix. With $N=A_{\mathrm{vh}}^{\mathrm{T}} Q_{\mathrm{vh}}^{-1} A_{\mathrm{vh}}$ and $\underline{l}=A_{\mathrm{vh}}^{\mathrm{T}} Q_{\mathrm{vh}}^{-1} \mathrm{vh}\left(\underline{t t}^{\mathrm{T}}\right)$, we can write

$$
\underline{\hat{\sigma}}=N^{-1} \underline{l} .
$$

After some simplifications [Amiri-Simkooei, 2007], the elements of the normal-matrix $N$ and the vector $\underline{l}$ can be expressed as

$$
\begin{aligned}
n_{r, l} & =\frac{1}{2} \operatorname{tr}\left(B^{\mathrm{T}} Q_{r} B Q_{t}^{-1} B^{\mathrm{T}} Q_{l} B Q_{t}^{-1}\right) \\
l_{r} & =\frac{1}{2} \underline{t}^{\mathrm{T}} Q_{t}^{-1} B^{\mathrm{T}} Q_{r} B Q_{t}^{-1} \underline{t}
\end{aligned}
$$

with $Q_{t}=B^{\mathrm{T}} Q B$ and tr the trace operator. Since $Q_{t}$ depends on the variance components $\underline{\sigma},(14)$ is again solved iteratively within each iteration of solving Eqn. (6), where $Q_{t}$ is computed with the estimates of the variance components $\sigma_{r}^{2}$ from the previous iteration.

In order to solve Eqn. (14), we therefore need to find a matrix $B$, whose columns are a basis of the null space of $A^{\mathrm{T}}$ such that $A^{\mathrm{T}} B=0$. There are infinitely many choices for $B$, all of which will lead to the same result $\underline{\hat{\sigma}}$. With $A=\left[\underline{e}_{R} \otimes I_{3 S P}\right]$, one solution is given by

$$
B=D \otimes I_{3 S P}
$$

with the differencing operator

$$
D=\left[\begin{array}{c}
-\underline{e}_{R-1}^{\mathrm{T}} \\
I_{R-1}
\end{array}\right] .
$$

That is, $\underline{t}=B^{\mathrm{T}} \underline{\underline{x}}$ in Eqn. (16) contains the between AC differenced orbits $\underline{x}_{r}-\underline{x}_{1}, r=\{2, \ldots, R\}$, and $\hat{\hat{\sigma}}$ is a function of only those differences.

With $Q_{r}=\operatorname{diag}\left(\underline{c}_{r}\right) \otimes I_{3 S P}$, we have

$$
B^{\mathrm{T}} Q_{r} B=\left(D^{\mathrm{T}} \operatorname{diag}\left(\underline{c}_{r}\right) D\right) \otimes I_{3 S P},
$$

and matrix $Q_{t}=B^{\mathrm{T}} Q B$ required in Eqns. (15) and (16) can be rewritten as

$$
Q_{t}=\left(\sum_{r=1}^{R} \sigma_{r}^{2} D^{\mathrm{T}} \operatorname{diag}\left(\underline{c}_{r}\right) D\right) \otimes I_{3 S P} .
$$

Consequently, Eqns. (15) and (16) are given by

$$
\begin{gathered}
n_{r, l}=\frac{3 S P}{2} \operatorname{tr}\left[D^{\mathrm{T}} \operatorname{diag}\left(\underline{c}_{r}\right) D\left(\sum_{q=1}^{R} \sigma_{q}^{2} D^{\mathrm{T}} \operatorname{diag}\left(\underline{c}_{q}\right) D\right)^{-1}\right. \\
\left.D^{\mathrm{T}} \operatorname{diag}\left(\underline{c}_{l}\right) D\left(\sum_{q=1}^{R} \sigma_{q}^{2} D^{\mathrm{T}} \operatorname{diag}\left(\underline{c}_{q}\right) D\right)^{-1}\right] \\
l_{r}=\frac{1}{2^{-}} t^{\mathrm{T}}\left\{\left[\left(\sum_{q=1}^{R} \sigma_{q}^{2} D^{\mathrm{T}} \operatorname{diag}\left(\underline{c}_{q}\right) D\right)^{-1} D^{\mathrm{T}} \operatorname{diag}\left(\underline{c}_{r}\right) D\right.\right. \\
\left.\left.\quad\left(\sum_{q=1}^{R} \sigma_{q}^{2} D^{\mathrm{T}} \operatorname{diag}\left(\underline{c}_{q}\right) D\right)^{-1}\right] \otimes I_{3 S P}\right\} \underline{t} .
\end{gathered}
$$

The blocks $D^{\mathrm{T}} \operatorname{diag}\left(\underline{c}_{r}\right) D$ used in this representation are only of dimension $R-1 \times R-1$ and therefore suitable for an efficient implementation. Using Eqns. (21) and (22) the variance components $\underline{\sigma}$ are estimated, $c f$. Eqn. (14), and used to compute the combined orbits, $c f$. Eqn. (12). 


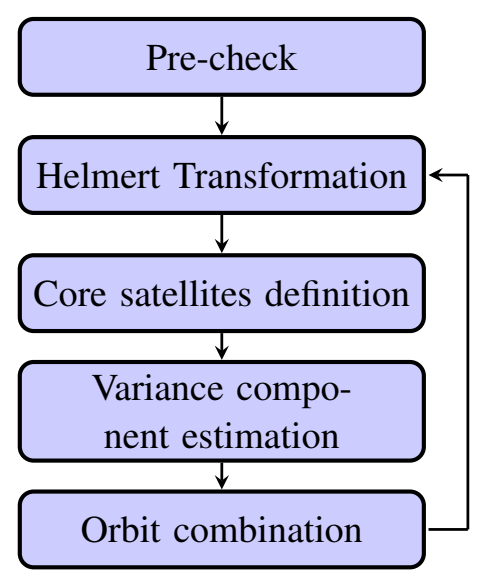

Figure 1: The flowchart represents the algorithm implemented to compute the orbit combination.

\subsection{Introducing constellation specific weights}

So far, we assumed that there is one variance component for each AC, see Eqns. (7) and (8), which translates into a weight per $\mathrm{AC}$ in the orbit combination in Eqn. (12). However, this assumption might be too simple for a multi-GNSS solution, in which the orbits of different constellations might be of different quality. We can therefore extend the above model by introducing a variance component ${ }_{g} \sigma_{r}^{2}, g \in\{1, \ldots, G\}$, for each $\mathrm{AC}$ and constellation, with $G$ the number of constellations. We define $g_{g} \underline{\bar{x}} \in \mathbb{R}^{3 S_{g} P R}$ as a vector similar to $\underline{\bar{x}}$ in Eqn. (6) that only contains all coordinates of a specific constellation with $S_{g}$ satellites. The system model then reads

$$
\left[\begin{array}{c}
1 \underline{\bar{x}} \\
\vdots \\
G \underline{\bar{x}}
\end{array}\right]=\left[\begin{array}{lll}
\underline{e}_{R} \otimes I_{3 S_{1} P} & & \\
& \ddots & \\
& & \underline{e}_{R} \otimes I_{3 S_{G} P}
\end{array}\right]\left[\begin{array}{c}
{ }_{1} \underline{x}_{\mathrm{c}} \\
\vdots \\
{ }_{G} \underline{x}_{\mathrm{c}}
\end{array}\right]+\left[\begin{array}{c}
1 \underline{\bar{\varepsilon}} \\
\vdots \\
G \underline{\bar{\varepsilon}}
\end{array}\right],
$$

where $g_{g} \underline{x}_{\mathrm{c}}$ and $g \underline{\bar{\varepsilon}}$ are the constellation specific versions of $\underline{x}_{\mathrm{c}}$ and $\underline{\bar{\varepsilon}}$. Assuming no correlations between different constellations, which is a natural consequence of the assumption in Section 2.1 that no correlations are present between the coordinates of different satellites, the covariance matrix $Q$ of the noise vector $\left[1 \underline{\bar{\varepsilon}}^{\mathrm{T}}, \ldots, G \underline{\bar{\varepsilon}}^{\mathrm{T}}\right]^{\mathrm{T}}$ is blockdiagonal

$$
Q=\left[\begin{array}{ccc}
1 Q & & \\
& \ddots & \\
& & { }_{G} Q
\end{array}\right]
$$

where ${ }_{g} Q$ only depends on ${ }_{g} \sigma_{r}^{2}$, with $r \in\{1, \ldots, R\}$. Therefore, the sets of parameters ${ }_{g} \underline{x}_{\mathrm{c}}$ and the variance components ${ }_{g} \sigma_{r}^{2}$ are mutually exclusive between the constellations, and the measurements $g \underline{\bar{x}}$ are uncorrelated so that the joint orbit combination and LSVCE can be computed separately for each constellation. It is important to note that this is only true within each iteration of the combination process and that the constellations are still connected through the common Helmert transformation in Eqn. (3).

\section{Combination steps}

The processing strategy of our orbit combination embedding the estimation scheme of Section 2 is shown in Figure 1. It is implemented in Python within the framework of the GeodeZYX toolbox [Sakic et al., 2019]. We can divide our orbit combination strategy into five major steps. 
(1) We start with a pre-check to identify and exclude satellites that appear in only one of the ACs' solutions or satellites with missing epochs. A first rough exclusion procedure is performed, in which the satellite coordinates are compared between ACs. If one AC provides an orbit for a satellite that is very far from the other ACs (e.g., threshold set at $500 \mathrm{~m}$ ), this solution is already excluded at this stage. A mean orbit is computed using the pre-checked orbits. (2) The second step consists of a Helmert transformation between this mean orbit and the ACs' solutions, which provides the seven parameters per AC that are used to align the orbits. (3) In the third step, a set of core satellites is defined. The idea is to select a subset of the orbits that are used in the VCE. The set of core satellites ensures that satellites are the same for all ACs when the variance components are computed and that outliers are detected and excluded to get proper estimates of the weights. The outlier detection is done by applying the Modified Z-Score [Iglewicz and Hoaglin, 1993] to the radial, along-track, and cross-track (RAC) components of each set of AC coordinates for all satellites. If one of the components is identified as an outlier for a satellite, this satellite is excluded from the set of core satellites. The detection threshold is chosen less strict for BeiDou and QZSS since the quality of the ACs' orbits for these systems is still not on the GPS, GLONASS, and Galileo level. (4) The fourth step is the estimation of the variance components as described in Section 2.3, using only the above defined set of core satellites. (5) With the variance components determined the final step is the orbit combination. As described in Eqn. (12) in Section 2.2, the inverse variances are normalized and then applied as weights to the ACs' orbits. The orbit combination is also provided for satellites that are not in the set of core satellites, where, if a solution is missing or excluded for one or more ACs for a specific satellite, the normalization is based only on the variances of the ACs that are included. This process is repeated as mentioned at the end of Section 2.1, starting with the Helmert transformation that is computed with respect to the combined orbits of the previous iteration. The iteration is terminated once the differences between the orbits of the current and previous iterations are smaller than the threshold of $10^{-6}$.

We can define three groups of the ACs' satellite orbits as input, where satellites excluded in the pre-check are not considered for the combination: (1) The first group contains the ACs' orbits of the core satellites that pass the outlier detection and are common among all ACs. They are the ones defining the weights of the combination. (2) The second group is used to compute the Helmert transformations. It includes the orbits of the core satellites but also of the satellites that are not available at all ACs. Satellites that are excluded as outliers for some of the ACs when defining the set of core satellites are also included for the remaining ACs. (3) The third group of satellite orbits is the largest group, which is used to compute the combined orbits. In addition to the second group, it also contains orbits of satellites that are detected as outliers for all ACs. They are kept in the orbit combination to provide a preferably complete solution.

\section{Results}

The two weighting approaches described in Section 2 are tested using products that are provided by the ACs to the IGS. We divide these products into two categories:

- Legacy ${ }^{2}$ : GPS and GLONASS products provided by the ACs within the regular IGS routine, for which the IGS provides a separate combination [Kouba, 2009; Johnston et al., 2017];

- $M G E X^{3}$ : products provided by the ACs to the Multi-GNSS Experiment and Pilot Project [Montenbruck et al., 2017]

Table 1 shows the ACs that contribute to the two processing lines and the constellations contained in the solutions. Some ACs provide both legacy and MGEX solutions. Except for GRG,

\footnotetext{
2 available in: ftp://igs.ign.fr/pub/igs/products/

${ }^{3}$ available in: ftp://igs.ign.fr/pub/igs/products/mgex/
} 

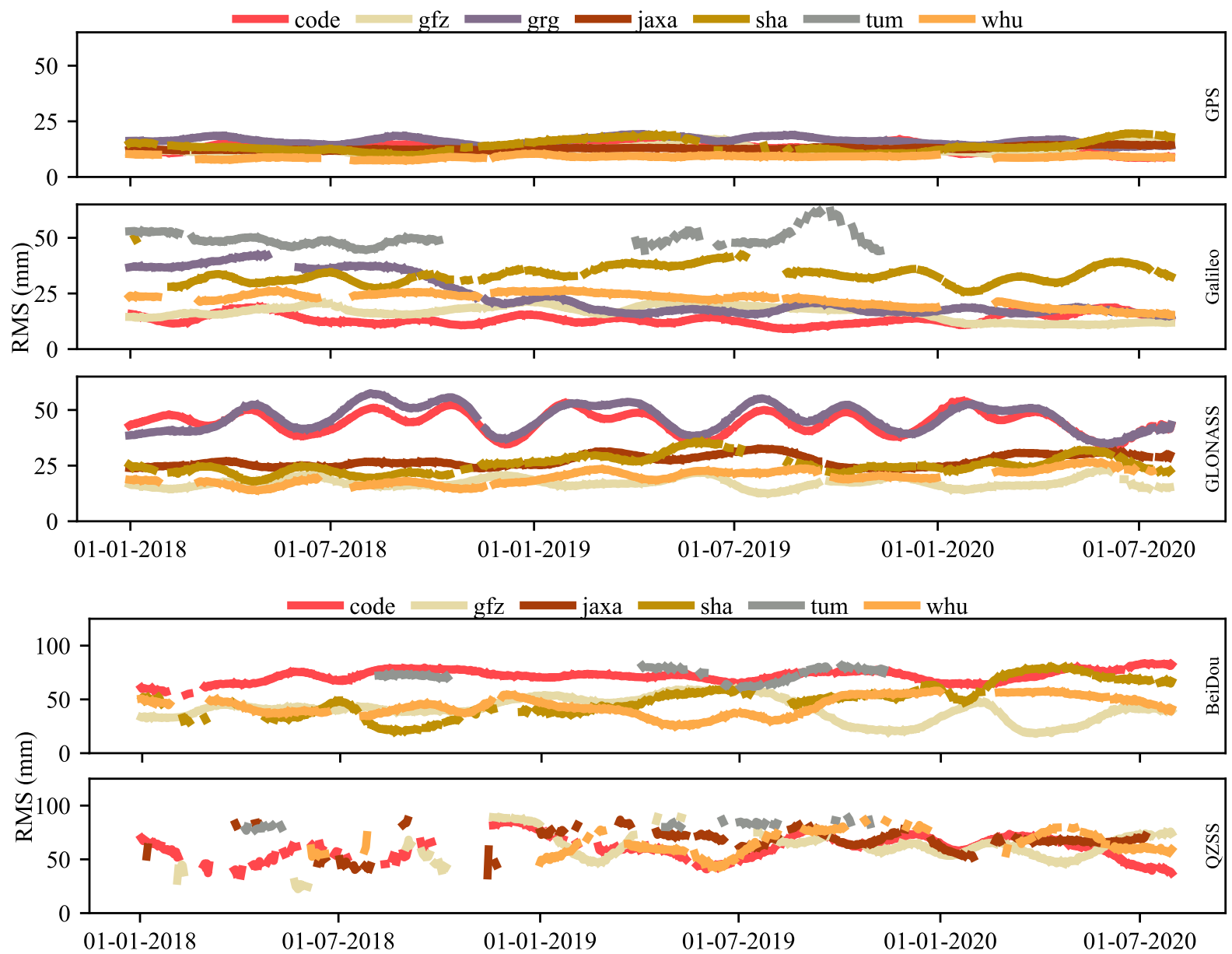

Figure 2: Constellation specific RMS differences between the individual AC orbits and the combined solution for AC plus constellation weighting. 


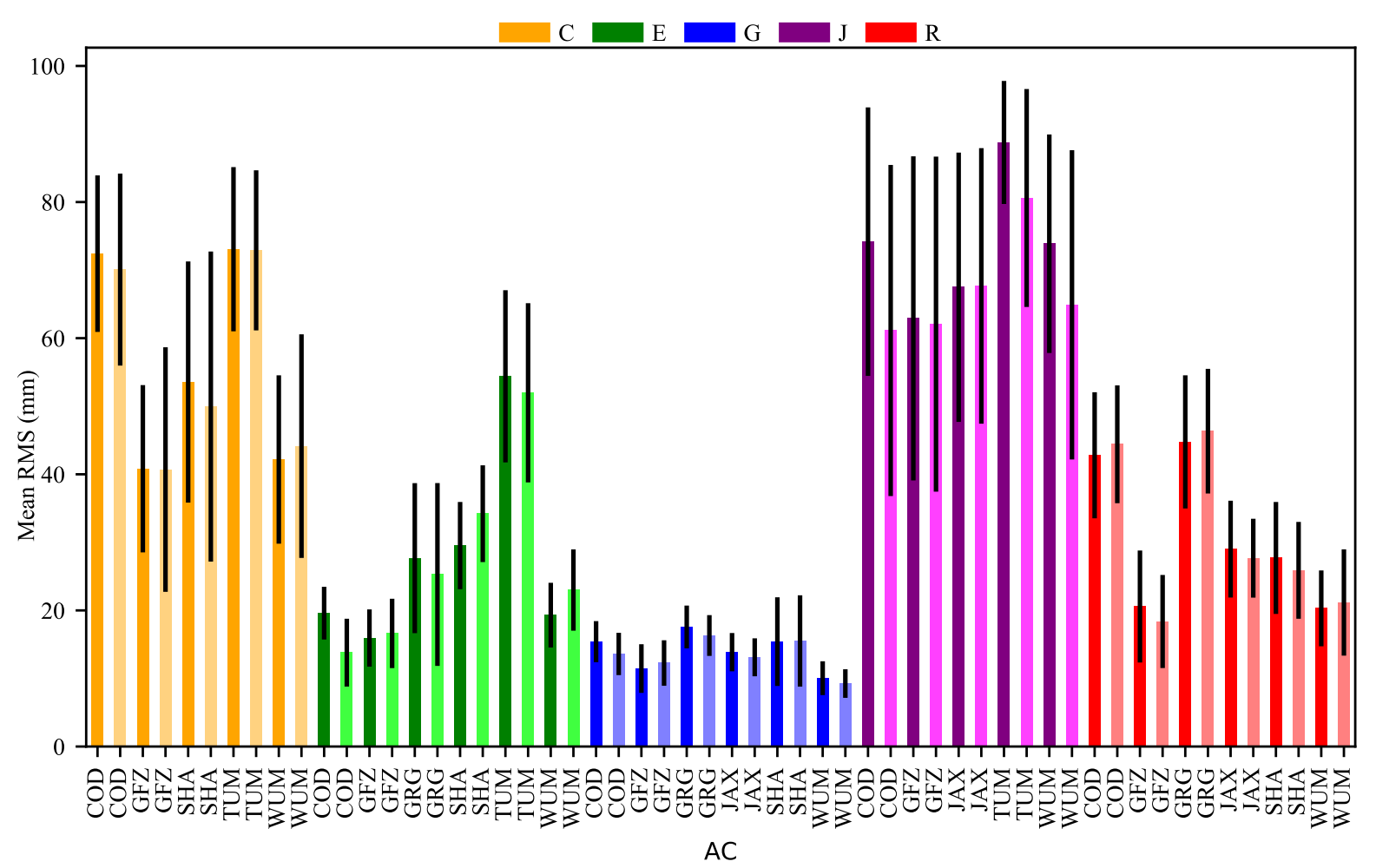

Figure 3: Comparison of the two combination approaches by means of the average RMS orbit differences between the AC orbits and the combined solution. The dark colors represent the ACs only weighting, and the light colors the AC plus constellation weighting.

these solutions are based on different processing setups and are therefore not identical. More information is provided in Mansur et al. [2020].

Two periods of processing are used for our analysis. For MGEX, we use orbit products between GPS week 1982 (January 2018) and GPS week 2116 (July 2020). GPS week 1930 (January 2017) until GPS week 2116 (August 2020) is considered with the legacy products.

\subsection{Differences between individual AC orbit solutions and combined orbits}

The results from the MGEX orbit combination are compared to the ACs' individual solutions. Figure 2 shows the time series of the RMS orbit differences with the AC plus constellation weighting approach. The RMS values are computed based on the formula in Kouba et al. [1994]. The smallest RMS values are obtained for GPS, and they are better than $25 \mathrm{~mm}$ for all ACs. QZSS shows the highest RMS values, which can reach around $80 \mathrm{~mm}$. This behavior is also noted by Sakic et al. [2020] who used an adaptation of the IGS legacy software to process the MGEX orbits. Similar results are provided by the ACC where another adaptation of the legacy software is used ${ }^{4}$. Moreover, for QZSS, especially in 2018, there are days without a combined solution due to missing data or high discrepancy between the satellite coordinates provided by the ACs yielding solutions with high RMS values. The days without a solution or with unreasonably high RMS values are not presented. One can note the gaps in Figure 2. In addition, the RMS analysis shows similar characteristics for some ACs for certain constellations, see, for example, the RMS values of GRG and CODE for GLONASS or of GFZ and WHU for most constellations. TUM has the most discrepant RMS values. Similar results are obtained with the ACs-only weighting approach, where the largest difference is obtained for QZSS with RMS values of around $90 \mathrm{~mm}$ instead of $80 \mathrm{~mm}$.

\footnotetext{
${ }^{4}$ http://acc.igs.org/mgex_experimental.html
} 


\begin{tabular}{l|ll|l}
\hline & Analysis Center & Abbr. & Constellations \\
\hline \multirow{5}{*}{ Legacy } & Natural Resources Canada & EMR/EMX & G + R \\
& Space geodesy team of the CNES & GRG & G + R \\
& European Space Agency & ESA/ESOC & G + R \\
& GeoForschungsZentrum Potsdam [Männel et al., 2020b] & GFZ & G + R \\
& Center for Orbit Determination in Europe [Dach et al., 2020] & CODE & G + R \\
& Jet Propulsion Laboratory & JPL & G \\
& NOAA/National Geodetic Survey & NGS & G \\
& Massachusetts Institute of Technology & MIT & G \\
& Scripps Institution of Oceanography & SIO & G \\
\hline \hline \multirow{5}{*}{ MGEX } & Space geodesy team of the CNES [Loyer et al., 2018; Katsigianni et al., 2018] & GRG & G + R + E \\
& Center for Orbit Determination in Europe [Prange et al., 2020a,b] & CODE & G + R + E + C + J \\
& GeoForschungsZentrum Potsdam [Uhlemann et al., 2015; Deng et al., 2016] & GFZ & G + R + E + C + J \\
& Japan Aerospace Exploration Agency & JAXA & G + R + J \\
& Shanghai Astronomical Observatory [Chen et al., 2012] & SHAO & G + R + E + C \\
& Technische Universität München [Selmke et al., 2018] & TUM & E + C + J \\
& Wuhan University [Guo et al., 2016] & WHU & G + R + E + C + J \\
\hline
\end{tabular}

Table 1: List of IGS ACs that provide orbit products to the legacy and MGEX lines. The following RINEX style constellation abbreviations are used: G - GPS, R - GLONASS, E - Galileo, C BeiDou, and J - QZSS.

Figure 3 shows the average of the daily RMS results per AC over the entire test period for both approaches. It is noted that TUM is excluded from the ACs-only weighting approach. As described in Section 3, our algorithm defines a list of core satellites, which are satellites provided by the majority of the ACs. Since TUM products do not contain GPS and GLONASS, this AC is excluded from the combination. However, a comparison of the TUM orbits with the combination is still provided. Comparing both strategies, only slight differences in the millimeter level have to be noted. The most significant differences are observed for QZSS with 13,11, and $8 \mathrm{~mm}$ for CODE, WHU, and TUM, respectively. The differences are less than $5 \mathrm{~mm}$ for GPS, followed by GLONASS, BeiDou, and Galileo.

Given that the Helmert transformation connects our ACs' orbits, as shown in Eqn. 3, we ensure small differences between the seven parameters obtained by our two strategies. On average, there are only differences in the millimeter level. These differences are related to the weighting scheme since, within both approaches, distinct mean orbits are computed and are used in the Helmert transformation.

\subsection{Weights}

Figures 4 and 5 show the weights used in the combination for the two approaches, and we can observe changes and trends of the ACs' weights over time. For the AC plus constellation weighting (Figure 4), GPS has the most consistent AC weights over the test period, followed by GLONASS and Galileo, while the weights for BeiDou and QZSS vary between the ACs. We might attribute this behavior to the number of satellites in the newer system, the different orbit types, or changes in the ACs' processing routines. We note that in our processing, we only consider the MEO satellites of the BeiDou constellation.

For the GPS constellation, WHU is the one with the weight majority, followed by GFZ, with an average of $28 \%$ and $21 \%$, respectively. We observe that since May 2020, CODE's weight has increased by $5 \%$ percentage points. Similar to GPS, for GLONASS, the most dominating weights are GFZ and WHU, and again CODE's weight increased at the end of the test period. For 

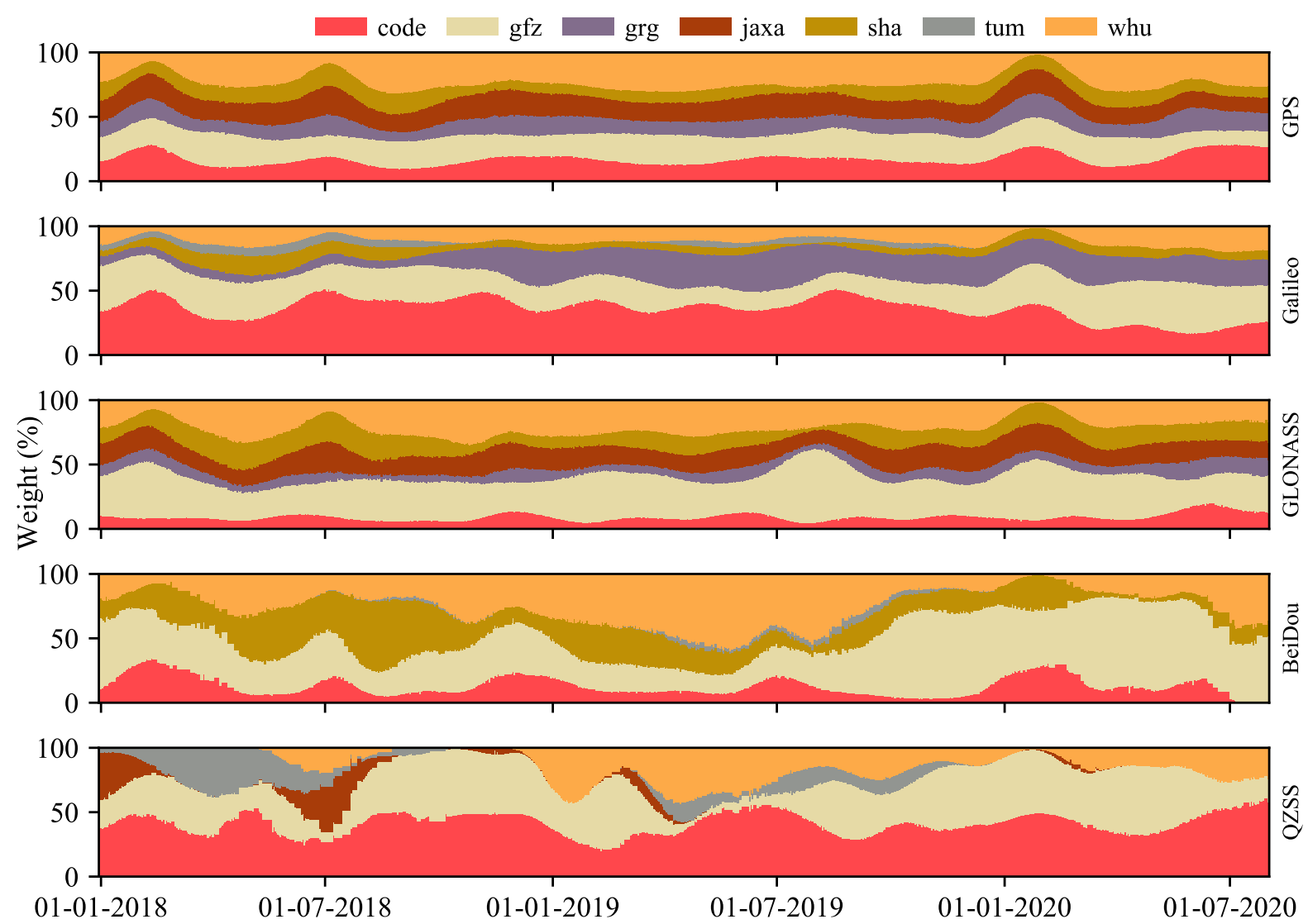

Figure 4: Constellation specific weights used in the orbit combination for the AC plus constellation weighting strategy.

Galileo, we can see an increase in the GRG weight at the end of 2018. We relate this to the time when GRG activated ambiguity fixing in their solution [Loyer et al., 2018]. In addition, for this constellation, CODE is the most stable AC in the years 2018 and 2019, but with a larger change in 2020, reducing the average weight of around $40 \%$ to $25 \%$. As mentioned before, BeiDou and QZSS have overall larger variations in the weights. For the Chinese system, the values are more or less evenly distributed among the ACs at the beginning of 2018. From April 2018, SHA had a big increase until January of 2019. In 2020, GFZ reaches an average weight of 50\% while WHU and SHA's weights decreased. From June 2020, CODE's BeiDou orbits are excluded from the combination. As mentioned before, our algorithm defines a set of core satellites commonly available at the majority of the ACs, on which the computation of the weights is based. This selection excluded the CODE BeiDou- 2 only solutions since the other ACs also provide BeiDou-3 solutions, which allows for a bigger set of core satellites without CODE. For QZSS, CODE got the major weight, with on average more than $30 \%$, followed by GFZ. Until July 2018, TUM had a significant weight, decreasing after this period while WHU's weights are more stable in 2019 and diminishing at the beginning of 2020.

The ACs-only weights shown in Figure 5 reveals that the values are more evenly distributed over the time of processing, but with clear advantages for GFZ and WHU. There is an increase of around 5\% for CODE and GRG, especially in the first half of 2020. However, one has to keep in mind that the weights are defined mainly based on the GPS, GLONASS, and Galileo since the satellites of these constellations are most represented in the core set. 


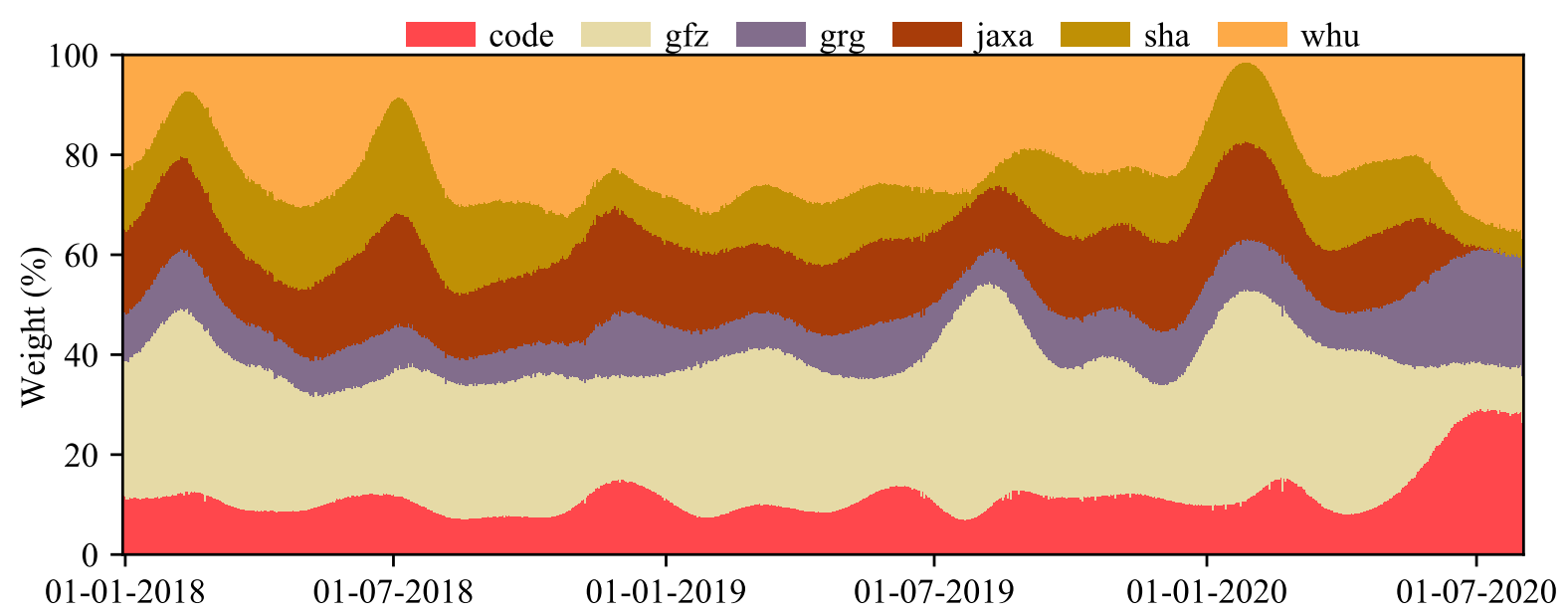

Figure 5: Weights used in the orbit combination for the ACs-only weighting strategy.

\subsection{Validation}

To provide a validation of our combination, two different validation steps have been performed. Firstly, we combine the orbit products provided within the IGS legacy chain using the ACs-only weighting approach. Secondly, the MGEX-based combined orbit solutions with both weighting strategies are validated using Satellite Laser Ranging (SLR).

\subsubsection{Combination of IGS legacy products}

Since only the constellations GPS or GLONASS are processed, we choose the ACs-only weighting approach. Figure 6 shows the GPS only RMS orbit differences between our combined solution, the ACs' orbits, and also the official IGS rapid and final orbits as reference. Similar to the ACC's official IGS results [Villiger, A., Dach, 2020], an agreement of around $15 \mathrm{~mm}$ is shown in our solution. Compared to the IGS rapid and final orbits, the RMS is $6 \mathrm{~mm}$ and $4 \mathrm{~mm}$, respectively, showing a very good agreement. Regarding the weights, CODE, on average, has the highest weight of $15 \%$, followed by NGS with $12 \%$ and JPL with $11 \%$. In addition, we observe that MIT's influence increased in 2020 when their weight raised from around 6\% in 2019 to $15 \%$ in 2020. For GLONASS, the RMS difference to the IGS final solution is around $12 \mathrm{~mm}$. The weights are more evenly distributed at the beginning of 2019, when ESA's weight decreased from an average of $55 \%$ to $30 \%$, while CODE and GRG show an increase of $10 \%$ each.

\subsubsection{SLR-based validation}

SLR measurements to the GNSS satellites provided by the International Laser Ranging Service (ILRS) establish an independent external validation of their orbits [Pearlman et al., 2019]. It is a tracking technique that depends on short laser pulses not susceptible to ionospheric delays, ambiguities, and other effects like tropospheric delays caused by water vapor that introduce errors in the observations. The SLR residuals are directly obtained from the difference between the geometric distance that comes from the GNSS orbits and the ITRF station coordinates, and the SLR measurements [Sośnica et al., 2015; Dach et al., 2019]. Currently, the satellites of Galileo, QZSS, and some of GLONASS and BeiDou are equipped with retro-reflectors and tracked by the ILRS. The number of SLR observations depends on the constellation and the ILRS priority list, with GLONASS and Galileo having in general more measurements than BeiDou and QZSS [Sośnica et al., 2020]. 


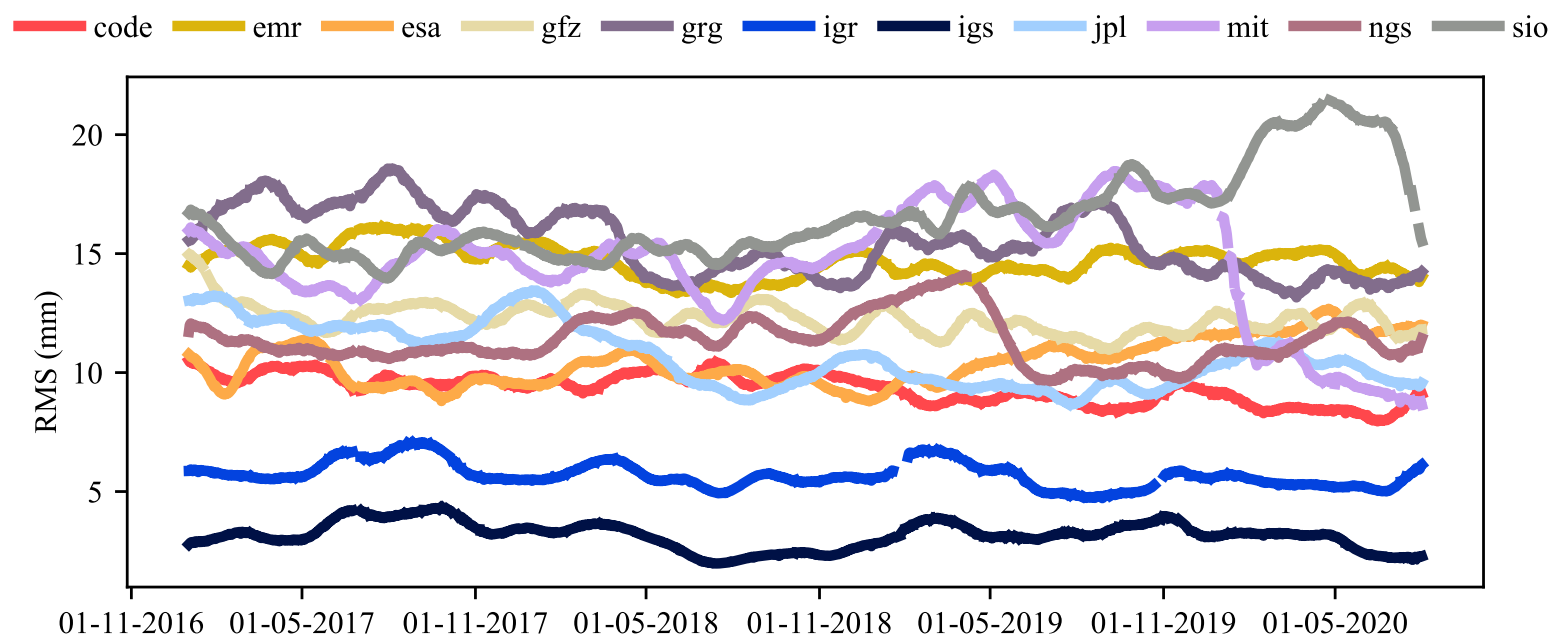

Figure 6: RMS orbit differences compared to the combined solution for the GPS only legacy processing. The IGS rapid (igr) and final (igs) solutions are included for validation.

The SLR residuals analysis is computed using the GFZ EPOS.P8 software [Gendt et al., 1998; Uhlemann et al., 2015] and confirms the similarity between both strategies, as the differences in the SLR residuals between both combination strategies are in the millimeter level. Regarding that, we show the results per Space Vehicle Number (SVN) from the AC plus constellation weighting approach in Figure 7. The constellation with the smallest standard deviation (STD) of the SLR residuals is Galileo with an average STD of $20 \pm 6 \mathrm{~mm}$, followed by GLONASS with $28 \pm 8 \mathrm{~mm}$, whereas $68 \pm 35 \mathrm{~mm}$ and $131 \pm 34 \mathrm{~mm}$ are obtained for BeiDou and QZSS, respectively.

In general, the offsets for Galileo are in the millimeter level, and the highest values are for the In-Orbit Validation (IOV) satellite group with negative offsets. However, the satellites with the minimum STD are E102 (IOV) and E209 with $14 \mathrm{~mm}$, which is from the Full Operational Capability (FOC) satellite group. The highest values of STD are for the satellites with eccentric orbits E201 and E202 with 45 and $35 \mathrm{~mm}$. For GLONASS, large positive offsets are observed for R855, R856, R858 of the block M+ with 36, 27, and $33 \mathrm{~mm}$ and for block M satellite R857 with $39 \mathrm{~mm}$. This behavior can also be noted in the preliminary GFZ repro3 results presented in Männel et al. [2020a] and the analysis of the experimental ACC combination shown in Sośnica et al. [2020]. Meanwhile, the lowest value of the standard deviation for GLONASS is obtained for R802 (K1B) with $15 \mathrm{~mm}$ and the highest for R730 (M) with $47 \mathrm{~mm}$. Unlike the other systems, for BeiDou, the smallest offsets and STD are observed using the ACs-only weighting approach. Using this strategy, the lowest offsets are for the satellites from BeiDou-3 with $7 \mathrm{~mm}$ for C207 (MEO) and $1 \mathrm{~mm}$ for $\mathrm{C} 208$ (MEO). Comparing the STD for these two satellites, the values are $80 \mathrm{~mm}$ and $125 \mathrm{~mm}$ for $\mathrm{C} 207$ and $\mathrm{C} 208$ when using the AC plus constellation weighting, whereas they are $49 \mathrm{~mm}$ and $34 \mathrm{~mm}$ when using the ACs-only weighting. The QZSS constellation has the most significant differences between both strategies. For all the satellites, the AC plus constellation weighting approach results in smaller offset and STD values. The minimum STD is observed for J001 (IQ) with $98 \mathrm{~mm}$ and the maximum for J003 (IIG) with $178 \mathrm{~mm}$. We note that for J002 (IIQ), we have the biggest difference of the STDs between both strategies with $41 \mathrm{~mm}$. The smallest offset is observed for J002 with $23 \mathrm{~mm}$, and the only satellite with negative offset values is J001. 


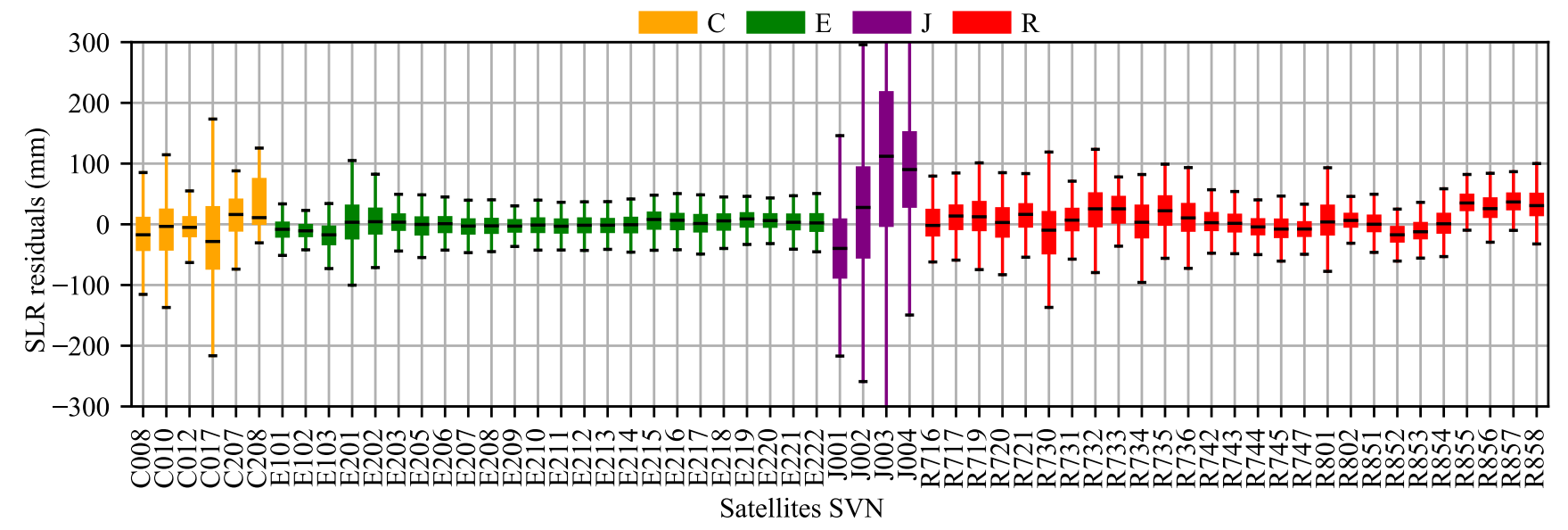

Figure 7: SLR Residuals of the combined orbits with AC plus constellation specific weighting. The black lines correspond to the medians, the boxes represent the Q1 to the Q3 quartile values, and the whiskers extend to 1.5 times the Q3-Q1 interquartile range.

\section{Conclusions}

In this study, we present and compare two approaches developed to combine GNSS orbits, where $\mathrm{AC}$ or $\mathrm{AC}$ and constellation specific weights are used. Section 2 describes the assumed functional and stochastic models, based on which a least-squares framework is formulated that jointly estimates the combined orbits and the weights used in the combination. Aiming for a reduced computational load, Eqns. (5) and (8) are derived to be easily implemented. Among the models' derivations, one important aspect is to find a basis matrix $B$ for the null space of $A^{\mathrm{T}}$. Given the large dimensions of the matrices when considering daily solutions with many satellites and several ACs, a direct computation may require heavy usage of the computer memory. Therefore, simplifications are needed for operational usage. With the Helmert transformation applied in Eqn. (5), a simple and structured form of the system matrix $A$ is found, enabling a closed form solution for $B$. The structure of this matrix $B$ again allows for an efficient implementation of the least-squares estimator of the variance components, which define the weights in the orbit combination, in which only matrices of small dimension have to be handled, see Eqns. 15 and 16. The method is initially developed for an ACs-only weighting. It was shown that the derivation of an AC plus constellation weighting scheme could be reduced to this problem since the weights and combined orbits can be solved individually for each constellation, and the constellations are only connected by a common Helmert transformation in each iteration. Another important topic of the proposed combination approach is the definition of a set of common core satellites, which are used to compute the weights of the combination. We propose a method based on the Modified Z-Score test that only selects satellites without outliers in the RAC components.

Both combination methods are tested using the MGEX ACs' orbits for a test period from January 2018 until August 2020. The analysis shows that using ACs-only or AC plus constellation weighting leads mostly to similar results where a significant RMS difference can be found only for QZSS. As expected, GPS has the smallest RMS with less than $25 \mathrm{~mm}$, while GLONASS and Galileo are below $50 \mathrm{~mm}$. For BeiDou and QZSS, the RMS reaches $80 \mathrm{~mm}$. Concerning the weights determined by the procedures, the AC plus constellation weighting scheme shows interesting results where one can notice the different behavior of the ACs for each system over time. For example, in the Galileo weights, there are significant changes for GRGS, CODE, and GFZ. Not just for the European system but likewise for BeiDou and QZSS, significant weight variations are observed, especially for WHU, GFZ, and CODE. 
A validation using products from the legacy IGS chain is performed. The RMS difference between the GPS-only combined orbit and the official IGS final and rapid orbits show an agreement of better than $4 \mathrm{~mm}$ and $6 \mathrm{~mm}$. These small differences for the standard GPS only case confirm the validity of the new combination method. As an external validation, the orbits generated by the two approaches are tested using SLR, assessing that the combined orbits can adequately represent the satellites' position. Within the SLR validation, Galileo showed the most accurate offsets, while QZSS has more discrepant values.

The results support the application of LSVCE to determine the weights for orbit combination. The implementation of the presented algorithm is sufficiently efficient to generate products on an operational basis, with a preliminary computational time of around ten minutes to process one day ${ }^{5}$. Judging the two strategies, even if the results are mostly similar, the AC plus constellation weighting reveals more information about the behavior of the ACs' solutions through the distribution of the weights for each constellation, so that for instance, problems and changes in the processing of each $\mathrm{AC}$ are easier visible. With more constellations becoming fully operational, several ACs are adapting their software to handle the new systems. It is therefore important to verify that an adaptation done for one system does not affect the others. Generally, from the experiments, one can see that the MGEX solutions submitted to the IGS are getting more stable over the years, and the ACs are putting efforts into providing products, including all constellations available. So far, the alignment with the ITRF is not implemented in the algorithm since there are no SINEX files provided by the ACs. In the next step of our study, we will include the alignment approach from Mansur et al. [2020] into our combination strategy.

\section{Author Contribution}

GM, PS, AB and BM designed the study. GM and PS implemented the algorithm. GM, PS, AB and $\mathrm{BM}$ wrote the paper. All the authors joined research discussion and gave their feedback for the paper writing.

\section{Data Availability}

The data used for this work are publicly and freely available on the International GNSS Service (IGS), for the regular products in: ftp://igs.ign.fr/pub/igs/products/. In the MGEX section of the IGS in: ftp://igs.ign.fr/pub/igs/products/mgex/. The combined products described in this study can be provided for free on demand.

We would like to thank the International Laser Ranging Service (ILRS), the International GNSS Service (IGS) and more particularly the MGEX Analysis Centers for providing their products. The PhD of Gustavo Mansur is funded by the Deutscher Akademischer Austauschdienst (DAAD, German Academic Exchange Service).

\section{References}

Amiri-Simkooei AR (2007) Least-squares variance component estimation - Theory and GPS applications. PhD thesis, NCG Nederlandse Commissie voor Geodesie

Beutler G, Kouba J, Springer T (1995) Combining the orbits of the IGS Analysis Centers. Bulletin Géodésique 69(4):200-222, DOI 10.1007/BF00806733, URL http: //link. springer.com/ $10.1007 / \mathrm{BF} 00806733$

\footnotetext{
${ }^{5}$ on a single computer equipped with a CPU Intel i5-4595 (4) @ 3.700GHz.
} 
Chen J, Wu B, Hu X, Li H (2012) SHA: The GNSS Analysis Center at SHAO. In: Sun J, Jiao W, Wu H, Shi C (eds) China Satellite Navigation Conference (CSNC) 2012 Proceedings, Springer Berlin Heidelberg, vol 243, pp 213-221, DOI 10.1007/978-3-642-29175-3_19

CSNO (2018) The BDS-3 Preliminary System Is Completed to Provide Global Services. URL http://en.beidou.gov.cn/WHATSNEWS/201812/t20181227_16837.html

Dach R, Sušnik A, Grahsl A, Villiger A, Schaer S, Arnold D, Prange L, Jäggi A (2019) Improving GLONASS orbit quality by re-estimating satellite antenna offsets. Advances in Space Research 63(12):3835-3847, DOI 10.1016/j.asr.2019.02.031

Dach R, Schaer S, Arnold D, Kalarus M, Prange L, Stebler P, Villiger A, Jaeggi A (2020) CODE final product series for the IGS. DOI 10.7892/boris.75876.4

Deng Z, Fritsche M, Nischan T, Bradke M (2016) Multi-GNSS ultra rapid orbit-, clock-and EOPproduct series. DOI http://doi.org/10.5880/GFZ

ESA (2016) Galileo begins serving the globe. URL http://www.esa.int/Our_Activities/ Navigation/Galileo_begins_serving_the_globe

Fritsche M (2016) Multi-GNSS orbit and clock combination: Preliminary results. EGU General Assembly Conference Abstracts

Gendt G, Dick G, Söhne W (1998) GFZ Analysis Center of IGS-Annual Report. IGS 1996 Annual Report pp 169-181

Griffiths J (2019) Combined orbits and clocks from IGS second reprocessing. Journal of Geodesy 93(2):177-195, DOI 10.1007/s00190-018-1149-8

Guo J, Xu X, Zhao Q, Liu J (2016) Precise orbit determination for quad-constellation satellites at Wuhan University: strategy, result validation, and comparison. Journal of Geodesy 90(2):143159, DOI 10.1007/s00190-015-0862-9

Iglewicz B, Hoaglin DC (1993) How to detect and handle outliers, vol 16. Asq Press

Johnston G, Riddell A, Hausler G (2017) The International GNSS Service, Springer International Publishing, Cham, pp 967-982. DOI 10.1007/978-3-319-42928-1_33

Katsigianni G, Loyer S, Perosanz F, Mercier F, Zajdel R, Sośnica K (2018) Improving Galileo orbit determination using zero-difference ambiguity fixing in a Multi-GNSS processing. Advances in Space Research DOI 10.1016/j.asr.2018.08.035

Kouba J (2009) A Guide to using international GNSS Service ( IGS ) Products. Geodetic Survey Division Natural Resources Canada Ottawa 6:34

Kouba J, Springer T (2001) New IGS Station and Satellite Clock Combination. GPS Solutions 4(4):31-36, DOI 10.1007/PL00012863

Kouba J, Mireault Y, Lahaye F (1994) IGS orbit/clock combination and evaluation. Tech. rep., Geodetic Survey Division, Geomatics Canada, Natural Resources Canada Ottawa, Canada

Loyer S, Perosanz F, Versini L, Katsigianni G, Mercier F, Mezerette A (2018) CNES/CLS IGS Analysis center: recent activities. In: IGS Workshop 2018, Wuhan

Männel B, Brandt A, Bradke M, Sakic P, Brack A, Nischan T (2020a) Status of IGS Reprocessing Activities at GFZ. DOI 10.1007/1345_2020_98 
Männel B, Brandt A, Nischan T, Brack A, Sakic P, Bradke M (2020b) GFZ final product series for the International GNSS Service (IGS). DOI 10.5880/GFZ.1.1.2020.002

Mansur G, Sakic P, Männel B, Schuh H (2020) Multi-constellation GNSS orbit combination based on MGEX products. Advances in Geosciences 50:57-64, DOI 10.5194/adgeo-50-57-2020

Montenbruck O, Steigenberger P, Prange L, Deng Z, Zhao Q, Perosanz F, Romero I, Noll C, Stürze A, Weber G, Schmid R, MacLeod K, Schaer S (2017) The Multi-GNSS Experiment (MGEX) of the International GNSS Service (IGS) - Achievements, prospects and challenges. Advances in Space Research 59(7):1671-1697, DOI 10.1016/j.asr.2017.01.011

Pearlman MR, Noll CE, Pavlis EC, Lemoine FG, Combrink L, Degnan JJ, Kirchner G, Schreiber U (2019) The ILRS: approaching 20 years and planning for the future. Journal of Geodesy 93(11):2161-2180, DOI 10.1007/s00190-019-01241-1

Prange L, Arnold D, Dach R, Kalarus S, Schaer S, Stebler P, Villiger A, Jäggi A (2020a) CODE product series for the IGS-MGEX project. DOI 10.7892/boris.75882.3

Prange L, Villiger A, Sidorov D, Schaer S, Beutler G, Dach R, Jäggi A (2020b) Overview of CODE's MGEX solution with the focus on Galileo. Advances in Space Research DOI https: //doi.org/10.1016/j.asr.2020.04.038

Sakic P, Mansur G, Kitpracha C, Ballu V (2019) The geodeZYX toolbox: a versatile Python 3 toolbox for geodetic-oriented purposes. V. 4.0. DOI 10.5880/GFZ.1.1.2019.002

Sakic P, Mansur G, Benjamin M (2020) A prototype for a Multi-GNSS orbit combination. In: European Navigation Conference 2020, pp 1-11, DOI https://doi.org/10.31223/osf.io/kxqsc

Selmke I, Duan B, Hugentobler U (2018) Status of the TUM MGEX orbit and clock products. In: IGS Workshop 2018, Wuhan

Sośnica K, Thaller D, Dach R, Steigenberger P, Beutler G, Arnold D, Jäggi A (2015) Satellite laser ranging to GPS and GLONASS. Journal of Geodesy 89(7):725-743, DOI 10.1007/ s00190-015-0810-8

Sośnica K, Zajdel R, Bury G, Bosy J, Moore M, Masoumi S (2020) Quality assessment of experimental IGS multi-GNSS combined orbits. GPS Solutions 24(2), DOI 10.1007/ s10291-020-0965-5

Springer TA, Beutler G (1993) Towards an official IGS orbit by combining the results of all IGS Processing Centers. In: Proceedings of the 1993 IGS Workshop, held March, pp 24-26

Teunissen PJ, Amiri-Simkooei AR (2008) Least-squares variance component estimation. Journal of Geodesy 82(2):65-82, DOI 10.1007/s00190-007-0157-x

Uhlemann M, Gendt G, Ramatschi M, Deng Z (2015) GFZ Global Multi-GNSS Network and Data Processing Results. In: International Association of Geodesy Symposia, vol 12, Springer, Cham, pp 673-679, DOI 10.1007/1345_2015_120

Villiger, A, Dach R (2020) International GNSS Service Technical Report 2019 (IGS Annual Report). Tech. rep., IGS Central Bureau and University of Bern, DOI 10.7892/boris.144003 